\title{
VENTILAÇÃO CONTROLADA COM PRESSÃO EXPIRATÓRIA FINAL POSITIVA EM SUÍNOS ANESTESIADOS COM ISOFLURANO OU HALOTANO E SUBMETIDOS A PNEUMOPERITÔNIO OU PNEUMOTÓRAX COM $\mathrm{CO}_{2}{ }^{*}$
}

\section{(Controlled ventilation with Positive End-Expiratory Pressure in isoflurane or halotane anesthetized pigs submitted to pneumoperitoneum or pneumothorax with $\mathrm{CO}_{2}$ )}

\author{
CUNHA, A.F. ${ }^{1}$; NATALINI, C.C. ${ }^{2}$; ALVES, S.D.L ${ }^{3}$; BOPP, S. ${ }^{4}$; FERREIRA, J.F. ${ }^{5}$; \\ POLYDORO, A.S. ${ }^{4}$; GUIMARÃES, L.D. ${ }^{4}$ \\ ${ }^{1}$ Anestesiologia Veterinária UFSM - afcunha2@bol.com.br; \\ ${ }^{2}$ Veterinary Clinical Sciences, School of Veterinary Medicine, LSU, Baton Rouge La. 70803.EUA; \\ ${ }^{3}$ Médica Veterinária, Anestesiologia Veterinária pela UFSM; \\ ${ }^{4}$ Médico Veterinário, Aluno do Programa de Pós-graduação em Medicina Veterinária da UFSM; \\ ${ }^{5}$ Médica Veterinária autônoma.
}

RESUMO - A homeostase hemodinâmica e respiratória nas vídeo cirurgias onde se emprega insuflação abdominal ou torácica requer métodos especiais de ventilação como a pressão expiratória final positiva (PEEP). Este experimento teve por objetivo avaliar a hemodinâmica e a oxigenação de suínos submetidos anestesia geral com isoflurano ou halotano onde foi realizado pneumoperitôneo a uma pressão constante de $15 \mathrm{mmHg}$ ou pneumotórax de $5 \mathrm{mmHg}$ com $\mathrm{CO}_{2}$ e ventilados com pressão expiratória zero ou $10 \mathrm{~cm}$ de $\mathrm{H}_{2} \mathrm{O}$. Foram estudadas as variáveis pressão arterial (sistólica, média e diastólica), freqüência cardíaca e respiratória, saturação de oxigênio na hemoglobina $\left(\mathrm{SpO}_{2}\right)$, volume corrente e minuto, eletrocardiografia e ainda pressão parcial de dióxido de carbono expirado. Onde foram observados valores de $\mathrm{SpO}_{2}$ mais elevados nos grupos submetidos a PEEP e maior hipotensão nos suínos anestesiados com halotano, nos permitindo concluir que quando comparado com o grupo que recebeu pressão expiratória final zero o uso de $10 \mathrm{~cm}$ de água de PEEP melhora a $\mathrm{SpO}_{2}$ e o isoflurano é superior ao halotano na manutenção da função cardiovascular de suínos submetidos à insuflação intra-abdominal ou intratorácica com $\mathrm{CO}_{2}$.

Palavras chave: vídeo-cirurgia, PEEP, ventilação artificial, ventilometria.

ABSTRACT - Hemodynamic and respiratory homeostasy in the video surgeries need special ventilatory methods as positive end-expiratory pressure (PEEP). This study aimed to investigate the hemodynamic and oxygenation effects in isoflurane or halotane anesthetized pigs, submitted to constant $15 \mathrm{~mm} \mathrm{Hg} \mathrm{CO}$ abdominal pressure or $5 \mathrm{~mm} \mathrm{Hg} \mathrm{CO}$ intrathoracic pressure of $10 \mathrm{~cm}$ of $\mathrm{H}_{2} \mathrm{O}$ of PEEP or $0 \mathrm{~cm}$ of $\mathrm{H}_{2} \mathrm{O}$ (ZEEP). Variables studied were arterial pressure (systolic, diastolic and mean) heart rate, respiratory rate, $\mathrm{SpO}_{2}$, tidal volume, minute volume, electrocardiogram and expiratory end-tidal carbon dioxide. Were observed improved in $\mathrm{SpO}_{2}$ with $10 \mathrm{~cm}$ of $\mathrm{H}_{2} \mathrm{O}$ PEEP and more hypotension in the halotane anesthetized group. We conclude that when compared to ZEEP group, $10 \mathrm{~cm} \mathrm{of} \mathrm{H}_{2} \mathrm{O}$ PEEP ventilation improves $\mathrm{SPO}_{2}$, while isoflurane is better to maintain the cardiovascular function to anesthetized pigs submitted to $15 \mathrm{~mm} \mathrm{Hg}$ intra-abdominal and $5 \mathrm{~mm} \mathrm{Hg}$ intratoracic pressure insufflations with $\mathrm{CO}_{2}$.

Key words: video surgery, PEEP, ventilation, ventilometry.

\footnotetext{
*Parte da Dissertação apresentada à Universidade Federal de Santa Maria (UFSM) para obtenção do Grau de Mestre em Medicina Veterinária.
} 


\section{Introdução}

As cirurgias vídeo-assistidas vêm sendo popularizadas por serem procedimentos minimamente invasivos (PAW e SACKIER, 1994) que desencadeiam pequeno estímulo doloroso no pósoperatório e menor período de recuperação (WALSH et al., 1999). De acordo com OHTSUKA et al. (1999) as vídeo-cirurgias realizadas na cavidade torácica ou abdominal requerem insuflação de Dióxido de Carbono $\left(\mathrm{CO}_{2}\right)$ para a visualização e manipulação adequada dos órgãos desencadeando alterações cardio-respiratórias (JONES et al., 1993) como hipotensão, hipoxemia, cianose, hipercarbia, aumento da freqüência respiratória, redução da saturação de oxigênio na hemoglobina $\left(\mathrm{SpO}_{2}\right)$ e aumento do dióxido de carbono expirado $\left(\mathrm{ETCO}_{2}\right)$ (TOGAL et al., 2002).

Para se manter a homeostasia respiratória em pacientes submetidos à insuflação intra-cavitária de $\mathrm{CO}_{2}$, pode-se utilizar a pressão expiratória final positiva (PEEP) (PADDLEFORD, 1999; BROCK et al., 2000), que consiste no aumento proposital da pressão nas vias aéreas entre os ciclos respiratórios, resultando em acúmulo de gás nos alvéolos, mantendo-os abertos, diminuindo as áreas de shunt alveolares e aumentando a capacidade residual funcional (PADDLEFORD, 1999).

Este experimento teve por objetivo avaliar a hemodinâmica e a oxigenação de suínos submetidos a pneumoperitôneo ou pneumotórax sob anestesia geral com isoflurano ou halotano e ventilados com zero ou $10 \mathrm{~cm}$ de $\mathrm{H}_{2} \mathrm{O}$ de pressão expiratória.

\section{Material e Métodos}

Foram estudados cinco suínos machos castrados, sem raça definida, com 50 a 70 dias de idade, pesando entre 15 e $35 \mathrm{~kg}$, alojados em baia coletiva onde receberam alimentação sólida duas vezes ao dia e água ad libitum. $\mathrm{O}$ desenho experimental seguiu o modelo de quadrado romano incompleto em medidas repetidas, oito tratamentos e cinco unidades experimentais.
Os procedimentos iniciaram com a contenção dos animais pelos membros pélvicos em um plano inclinado, onde a cabeça foi mantida para baixo, seguido do acoplamento de uma máscara facial para indução anestésica diretamente com anestésico volátil. Assim que foi observado relaxamento muscular sondou-se a traquéia e conectou-se os pacientes ao circuito anestésico circular semi-fechado e aos sensores dos monitores. Em seguida a veia marginal da orelha foi cateterizada para instituição de fluidoterapia com solução de Ringer-lactato na dose de $10 \mathrm{ml} / \mathrm{kg} / \mathrm{hora}$. Os suínos foram anestesiados com halotano ou isoflurano e mantidos sob ventilação mecanicamente controlada com valores de volume minuto padronizados com base nos valores de $\mathrm{ETCO}_{2}$, para manter os animais em normocarbia $\left(\mathrm{ETCO}_{2}\right.$ entre 35 e $\left.45 \mathrm{~mm} \mathrm{Hg}\right)$.

Dez minutos após o inicio da ventilação mecânica (denominado como tempo basal) puncionou-se o abdômen nos animais que foram submetidos a pneumoperitôneo) com uma agulha de Veress na cicatriz umbilical e insuflou-se $\mathrm{CO}_{2}$ a uma pressão intra-abdominal constante de $15 \mathrm{~mm} \mathrm{Hg}$, durante oitenta minutos. Nos animais que foram submetidos a pneumotórax, após o tempo basal, realizou-se punção no oitavo espaço intercostal e insuflação $\mathrm{CO}_{2}$ a uma pressão constante de 5 $\mathrm{mm} \mathrm{Hg}$ também durante 80 minutos.

Os 5 suínos foram anestesiados quatro vezes, com intervalo de três dias entre cada procedimento, para que fossem submetidos a pneumoperitôneo, sob anestesia geral com halotano ou isoflurano e ventilação mecânica com ou sem PEEP e depois foram submetidos a pneumotórax para os mesmos procedimentos. A PEEP foi obtida pela conexão da saída de ar expirado do ventilador mecânico a um recipiente contendo uma coluna de $10 \mathrm{~cm}$ de água, através de um tubo corrugado.

A ventilação mecânica perdurou oitenta minutos e foi dividida em dois momentos onde foi realizado 40 minutos de PEEP seguidos de 40 minutos de ZEEP na metade dos procedimentos e o inverso na outra metade, ou seja, 40 minutos de ZEEP seguidos de 40 minutos de PEEP, conforme FIGURA 1. 
Ventilação controlada com pressão expiratória final positiva em suínos anestesiados com isoflurano...

FIGURA1 - DISTRIBUIÇÃO DOS GRUPOS DE SUÍNOS SUBMETIDOS A PNEUMOTÓRAX OU PNEUMOPERITÔNEO, ANESTESIADOS COM ISOFLURANO OU HALOTANO, CONFORME DIVISÃO DOS TEMPOS E INÍCIO DOS MODOS DE VENTILAÇÃO (PRESSÃO EXPIRATÓRIA FINAL DE $10 \mathrm{CM} \mathrm{H} \mathrm{H}_{2} \mathrm{O}$ - PEEP10 OU PRESSÃO EXPIRATÓRIA FINAL ZERO - ZEEP). (SANTA MARIA, RS 2002).

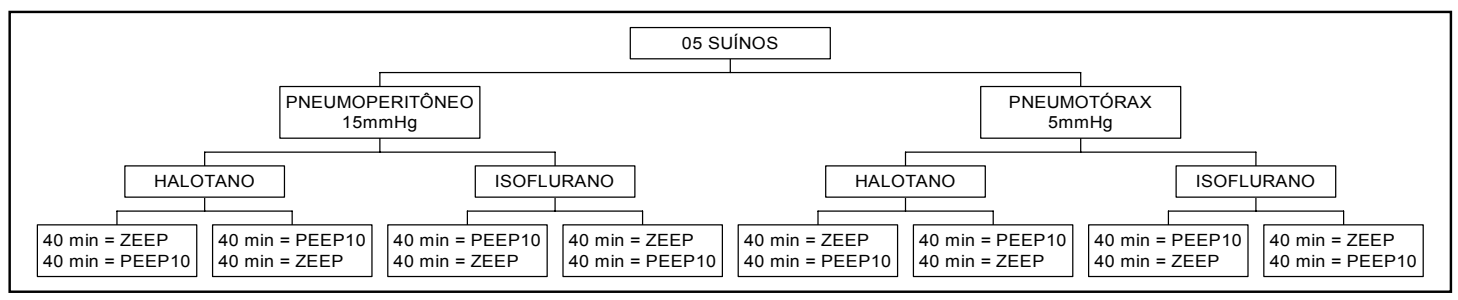

As variáveis estudadas foram: pressão arterial sistólica (PAS), média (PAM) e diastólica (PAD) com utilização de um monitor oscilométrico não invasivo, saturação de oxigênio na hemoglobina $\left(\mathrm{SpO}_{2}\right)$ e freqüência cardíaca $(\mathrm{FC})$ pela oximetria de pulso, freqüência respiratória (FR), volume corrente (VC) e volume minuto (VM) através de um ventilômetro digital, pressão parcial de dióxido de carbono expirado $\left(\mathrm{ETCO}_{2}\right)$ pela capnometria e ritmo cardíaco através da eletrocardiografia.

As médias foram tabuladas e submetidas à análise de variância, com posterior teste Tukey nos casos onde foi observada diferença estatisticamente significativa, ou seja, $p<0,05$, utilizando-se o programa estatístico SAS, 1997.

\section{Resultados e Discussão}

Mesmo com utilização de ventilação mecanicamente controlada e administração de oxigênio a $100 \%$, foram observados vários momentos onde a $\mathrm{SpO}_{2}$ encontrava-se abaixo de 95\%, principalmente nos grupos que não receberam PEEP e nos suínos submetidos a pneumotórax, conforme TABELAS 1 e 3. Este fato pode ser explicado por dois motivos principais: o aumento da pressão intra-abdominal (PIA), que projeta o diafragma em direção aos pulmões, alterando o mecanismo respiratório, por redução do volume corrente (BAILEY e PABLO, 1998) e concomitante diminuição da capacidade residual funcional (LOECKINGER et al., 2000), dificultando as trocas gasosas (BAILEY e PABLO, 1998), agravados pela distensão abdominal e pressão sobre os grandes vasos abdominais, que gera redução do retorno venoso e diminuição do débito cardíaco (BAILEY e PABLO, 1998), levando à redução da perfusão periférica e aumento da extração de $\mathrm{O}_{2}$ pelos tecidos (BROCK et al., 2000).

$\mathrm{O}$ segundo fator é a utilização do $\mathrm{CO}_{2}$ para manutenção da PIA, pois este gás possui taxa de solubilidade sanguínea cerca de 20 vezes maior que $\circ \mathrm{O}_{2}$ e alta afinidade com a água (GUYTON, 1991), fatores que lhe conferem grande facilidade para transpor a barreira celular causando hipercarbia, principalmente pela diferença entre concentrações da cavidade insuflada e valores sistêmicos de $\mathrm{CO}_{2}$, devendo ser removido pelo processo da ventilação pulmonar (GUYTON, 1991), elevando a $\mathrm{ETCO}_{2}$ e reduzindo a $\mathrm{SpO}_{2}$.

Assim como em nosso experimento, BROCK et al. (2000) observaram redução da $\mathrm{SPO}_{2}$ após insuflação intrapleural com $\mathrm{CO}_{2}$ a uma pressão constante de 5 e 15 $\mathrm{mm} \mathrm{Hg}$. Este autor relata que a presença de pneumotórax agrava a dificuldade na eliminação do $\mathrm{CO}_{2}$, devido ao aumento da pressão intratorácica, que faz a relação ventilação alveolar/perfusão $\left(V_{A} / Q\right)$ diminuir, ou seja, aumenta o número de alvéolos sem ventilação, diminuindo a $\mathrm{PCO}_{2}$ alveolar o que dificulta a eliminação do $\mathrm{CO}_{2}$ (GUYTON, 1991). 
146

CUNHA, A.F. et al.

TABELA 1 - FUNÇÃO HEMODINÂMICA DE SUÍNOS SUBMETIDOS A PNEUMOPERITÔNEO COM 15 mmHg DE $\mathrm{CO}_{2}$, ANESTESIADOS COM HALOTANO OU ISOFLURANO E VENTILADOS COM PRESSÃO EXPIRATÓRIA FINAL POSITIVA DE $10 \mathrm{~cm}$ DE $\mathrm{H}_{2} \mathrm{O}$ (PEEP) OU PRESSÃO EXPIRATÓRIA FINAL ZERO (ZEEP) - SANTA MARIA - RS/2002.

\begin{tabular}{|c|c|c|c|c|c|c|}
\hline Variáveis & Grupos & Valores basais & $\begin{array}{l}10 \text { min. de } \\
\text { ventilação }\end{array}$ & $\begin{array}{l}20 \text { min. de } \\
\text { ventilação }\end{array}$ & $\begin{array}{c}30 \text { mim } \\
\text { ventilação }\end{array}$ & $\begin{array}{c}40 \text { min. } \\
\text { Ventilação }\end{array}$ \\
\hline PAS & $\mathrm{H} / \mathrm{PEEP}$ & $75,75 \pm 3,74$ & $71,25 \pm 8,96^{\mathrm{B}}$ & $80,00 \pm 6,16^{\mathrm{BC}}$ & $75,75 \pm 6,99$ & $73,00 \pm 9,76$ \\
\hline \multirow[t]{3}{*}{$\mathrm{MmHg}$} & H / ZEEP & $75,75 \pm 3,74$ & $79,25 \pm 5,56^{\mathrm{AB}}$ & $68,50 \pm 6,56^{\mathrm{C}}$ & $75,50 \pm 13,87$ & $72,75 \pm 4,57$ \\
\hline & I / PEEP & $86,25 \pm 16,24^{\mathrm{ab}}$ & $100,00 \pm 14,40^{\mathrm{Aa}}$ & $90,75 \pm 13,25^{\mathrm{ABab}}$ & $79,75 \pm 5,50^{\mathrm{ab}}$ & $78,75 \pm 5,56^{b}$ \\
\hline & I / ZEEP & $86,25 \pm 16,25$ & $92,25 \pm 21,87^{A B}$ & $94,00 \pm 14,99^{\mathrm{AB}}$ & $88,25 \pm 14,55$ & $91,75 \pm 13,05$ \\
\hline PAM & H / PEEP & $50,00 \pm 3,74$ & $49,25 \pm 9,81$ & $52,00 \pm 4,90$ & $48,00 \pm 8,60$ & $48,50 \pm 5,92$ \\
\hline \multirow[t]{3}{*}{$\mathrm{mmHg}$} & H / ZEEP & $50,00 \pm 3,74$ & $53,75 \pm 4,27$ & $48,50 \pm 5,26$ & $42,00 \pm 4,83$ & $51,00 \pm 3,16$ \\
\hline & I / PEEP & $62,00 \pm 11,28^{\mathrm{ab}}$ & $75,25 \pm 11,59^{a}$ & $62,25 \pm 11,59^{\mathrm{ab}}$ & $56,00 \pm 8,37^{\mathrm{ab}}$ & $56,50 \pm 8,50^{\mathrm{ab}}$ \\
\hline & I / ZEEP & $62,00 \pm 11,29$ & $71,50 \pm 22,22$ & $60,50 \pm 13,40$ & $59,50 \pm 15,76$ & $55,25 \pm 11,87$ \\
\hline PAD & H / PEEP & $38,25 \pm 7,68$ & $34,25 \pm 6,80$ & $35,75 \pm 5,38$ & $37,50 \pm 3,79$ & $34,25 \pm 4,50$ \\
\hline \multirow[t]{3}{*}{$\mathrm{mmHg}$} & H / ZEEP & $38,25 \pm 7,68$ & $34,00 \pm 2,16$ & $33,25 \pm 5,12$ & $31,50 \pm 2,52$ & $35,75 \pm 3,86$ \\
\hline & I / PEEP & $42,50 \pm 1,73^{\mathrm{ab}}$ & $61,75 \pm 13,94^{a}$ & $49,75 \pm 8,42^{\mathrm{ab}}$ & $42,50 \pm 4,65^{\mathrm{ab}}$ & $44,75 \pm 7,89^{b}$ \\
\hline & I / ZEEP & $42,50 \pm 1,74$ & $60,50 \pm 21,52$ & $48,50 \pm 12,45$ & $44,75 \pm 14,86$ & $42,25 \pm 10,72$ \\
\hline $\mathrm{FC}$ & H / PEEP & $121,50 \pm 14,29^{a}$ & $77,25 \pm 2,06^{b}$ & $78,75 \pm 2,99^{b}$ & $82,00 \pm 3,37^{b}$ & $80,50 \pm 2,38^{b}$ \\
\hline \multirow[t]{3}{*}{ BPM } & H / ZEEP & $121,50 \pm 14,29^{a}$ & $83,25 \pm 3,69^{b}$ & $80,00 \pm 2,45^{b}$ & $78,50 \pm 2,52^{\mathrm{b}}$ & $78,50 \pm 3,11^{\mathrm{b}}$ \\
\hline & I / PEEP & $124,50 \pm 23,46^{a}$ & $99,75 \pm 24,05^{b}$ & $103,50 \pm 24,28^{b}$ & $100,00 \pm 19,08^{b}$ & $100,00 \pm 21,56^{b}$ \\
\hline & I / ZEEP & $124,50 \pm 23,47^{a}$ & $95,50 \pm 21,76^{b}$ & $99,50 \pm 20,81^{b}$ & $97,75 \pm 20,52^{b}$ & $105,50 \pm 27,21^{b}$ \\
\hline \multirow[t]{4}{*}{ V\% } & $\mathrm{H} / \mathrm{PEEP}$ & $1,50 \pm 0,00^{\mathrm{Aa}}$ & $0,88 \pm 0,48^{\mathrm{Ab}}$ & $0,88 \pm 0,25^{\mathrm{Ab}}$ & $0,88 \pm 0,25^{A b}$ & $0,88 \pm 0,25^{\mathrm{Ab}}$ \\
\hline & $\mathrm{H} / \mathrm{ZEEP}$ & $1,50 \pm 0,00^{\mathrm{Aa}}$ & $1,00 \pm 0,00^{\mathrm{Ab}}$ & $0,89 \pm 0,13^{A b c}$ & $0,75 \pm 0,20^{\mathrm{Ac}}$ & $0,57 \pm 0,41^{\mathrm{AC}}$ \\
\hline & I / PEEP & $2,88 \pm 0,48^{B}$ & $2,75 \pm 0,65^{\mathrm{B}}$ & $2,63 \pm 0,48^{\mathrm{B}}$ & $2,88 \pm 0,25^{\mathrm{B}}$ & $2,63 \pm 0,25^{\mathrm{B}}$ \\
\hline & I / ZEEP & $2,88 \pm 0,49^{\mathrm{Ba}}$ & $2,50 \pm 0,41^{\mathrm{Bab}}$ & $3,00 \pm 0,48^{\mathrm{Bb}}$ & $3,00 \pm 0,48^{\mathrm{Bb}}$ & $2,75 \pm 0,29^{\mathrm{Bab}}$ \\
\hline
\end{tabular}

Valores expressos em média \pm desvio padrão onde: ${ }^{A B C}=$ diferenças entre grupos ao longo dos momentos, ${ }^{\mathrm{abc}}=$ diferenças entre tempos dentro de cada grupo, onde $p<0,05 ;$ PAS = Pressão arterial sistólica, PAM = Pressão arterial média, $P A D=$ Pressão arterial diastólica, $F C=$ Freqüência cardíaca, $V \%=$ Vaporização do gás anestésico, H/PEEP = Grupo halotano + PEEP, H/ZEEP $=$ Grupo halotano + ZEEP, I/PEEP = Isoflurano + PEEP, I/ZEEP = Isoflurano + ZEEP.

$O$ uso da PEEP aumenta a relação $V_{A} / Q$, refletindo na quantidade de alvéolos ventilados, melhorando as trocas gasosas entre alvéolos e circulação sanguínea (GUYTON, 1991; LOECKINGER et al., 2000). Nos pacientes que receberam PEEP de $10 \mathrm{~cm} \mathrm{H} \mathrm{H}_{2} \mathrm{O}$ foi observado aumento na $\mathrm{SpO}_{2}$, mais evidente no grupo submetido a pneumotórax, explicado por MALLEY (1990), LOECKINGER et al. (2000) que afirmam que a PEEP aumenta a eliminação de $\mathrm{CO}_{2}$ e melhora a oxigenação por dois motivos principais: maior número de alvéolos ventilados, diminuindo as áreas de "shunts" alveolares e aumento da capacidade residual funcional.

LOECKINGER et al. (2000) e MALLEY (1990) afirmam que pneumoperitôneo com $\mathrm{CO}_{2}$ associado à respiração assistida com PEEP reduz o débito cardíaco, diminui o retorno sanguíneo para o coração e descreve dois mecanismos de indução do decréscimo do débito cardíaco pelo uso de PEEP. O primeiro através do aumento da pressão intratorácica no momento da expansão dos pulmões, comprimindo grandes vasos torácicos, diminuindo o retorno venoso e o segundo pela compressão dos vasos pulmonares provocando aumento na resistência vascular e aumento da pressão no ventrículo direito, prejudicando a contratilidade do miocárdio, que segundo TORRES e BONASSA (2002) será proporcional a PEEP empregada.

No que se refere à pressão arterial, foi observado hipotensão em todos os grupos, tanto nos suínos submetidos a pneumotórax quanto a pneumoperitôneo, efeito também observado por MALLEY (1990) e LOECKINGER et al. (2000) que relatam o aumento da pressão intra-abdominal como causa de hipotensão e BROCK et al. (2000) que afirmam que o pneumotórax tem efeito sobre o débito cardíaco e a pressão arterial, também causando hipotensão. 
Ventilação controlada com pressão expiratória final positiva em suínos anestesiados com isoflurano...

TABELA 2 - FUNÇÃO RESPIRATÓRIA DE SUÍNOS SUBMETIDOS A PNEUMOPERITÔNEO COM $15 \mathrm{mmHg}$ DE $\mathrm{CO}_{2}$, ANESTESIADOS COM HALOTANO OU ISOFLURANO E VENTILADOS COM PRESSÃO EXPIRATÓRIA FINAL POSITIVA DE $10 \mathrm{~cm}$ DE $\mathrm{H}_{2} \mathrm{O}$ (PEEP) OU PRESSÃO EXPIRATÓRIA FINAL ZERO (ZEEP) SANTA MARIA - RS/2002.

\begin{tabular}{|c|c|c|c|c|c|c|}
\hline Variáveis & Grupos & Valores basais & $\begin{array}{l}10 \text { min. de } \\
\text { ventilação }\end{array}$ & $\begin{array}{l}20 \text { min. de } \\
\text { ventilação }\end{array}$ & $\begin{array}{l}30 \text { min. de } \\
\text { ventilação }\end{array}$ & $\begin{array}{l}40 \text { min. de } \\
\text { ventilação }\end{array}$ \\
\hline $\mathrm{SPO}_{2}$ & H / PEEP & $98,50 \pm 2,38$ & $96,5 \pm 2,65$ & $97,00 \pm 2,94$ & $97,25 \pm 2,50$ & $97,25 \pm 3,10$ \\
\hline \multirow[t]{3}{*}{$\%$} & H / ZEEP & $98,50 \pm 2,38$ & $95,25 \pm 5,68$ & $95,00 \pm 4,69$ & $95,50 \pm 4,20$ & $97,00 \pm 2,16$ \\
\hline & I / PEEP & $97,50 \pm 1,91$ & $95,75 \pm 2,22$ & $97,75 \pm 1,26$ & $97,50 \pm 1,29$ & $97,50 \pm 1,73$ \\
\hline & I / ZEEP & $97,50 \pm 1,92$ & $96,50 \pm 3,11$ & $97,00 \pm 2,71$ & $97,25 \pm 1,71$ & $97,75 \pm 1,89$ \\
\hline FR & H / PEEP & $19,75 \pm 2,06^{\mathrm{Ba}}$ & $15,00 \pm 3,92^{\mathrm{ab}}$ & $13,00 \pm 2,16^{\mathrm{ab}}$ & $13,25 \pm 1,26^{\mathrm{ab}}$ & $12,75 \pm 2,06^{b}$ \\
\hline \multirow[t]{3}{*}{ MPM } & H / ZEEP & $19,75 \pm 2,06^{\mathrm{Ba}}$ & $13,75 \pm 5,56^{\mathrm{ab}}$ & $11,50 \pm 1,00^{b}$ & $9,50 \pm 5,69^{b}$ & $11,00 \pm 1,41^{b}$ \\
\hline & I/ PEEP & $23,00 \pm 8,52^{\mathrm{Aa}}$ & $16,00 \pm 7,39^{b}$ & $11,00 \pm 3,92^{b}$ & $15,00 \pm 3,37^{b}$ & $13,25 \pm 2,22^{b}$ \\
\hline & I / ZEEP & $23,00 \pm 8,53^{A}$ & $19,75 \pm 7,54$ & $11,25 \pm 3,30$ & $13,75 \pm 5,74$ & $14,00 \pm 6,06$ \\
\hline VC & H / PEEP & $149,75 \pm 32,94^{b}$ & $165,50 \pm 33,75^{a b}$ & $171,75 \pm 27,57^{a}$ & $180,50 \pm 30,75^{a}$ & $176,75 \pm 26,42^{a b}$ \\
\hline \multirow[t]{3}{*}{$\mathrm{ml}$} & H / ZEEP & $149,75 \pm 32,94^{b}$ & $209,00 \pm 35,05^{a}$ & $190,25 \pm 42,07^{a}$ & $195,50 \pm 36,43^{a}$ & $183,75 \pm 53,20^{a}$ \\
\hline & I / PEEP & $165,75 \pm 68,05$ & $197,50 \pm 53,42$ & $216,00 \pm 32,34$ & $186,00 \pm 23,34$ & $160,75 \pm 14,66$ \\
\hline & I / ZEEP & $\begin{array}{c}165,75 \pm 68,06 \\
2990,25 \pm\end{array}$ & $184,00 \pm 57,53$ & $230,00 \pm 28,72$ & $223,00 \pm 41,03$ & $189,25 \pm 83,37$ \\
\hline VM & H / PEEP & $\begin{array}{c}889,69^{\mathrm{a}} \\
2990,25 \pm\end{array}$ & $1993,50 \pm 240,02^{b}$ & $2316,75 \pm 354,99^{\mathrm{ab}}$ & $2477,50 \pm 459,20^{a b}$ & $2073,00 \pm 159,54^{b}$ \\
\hline \multirow[t]{3}{*}{$\mathrm{ml} / \mathrm{min}$} & H / ZEEP & $889,69^{a}$ & $2347,75 \pm 587,82^{a}$ & $2274,50 \pm 560,72^{a}$ & $2317,00 \pm 551,77^{a}$ & $2086,00 \pm 822,97^{a}$ \\
\hline & I / PEEP & $1968,75 \pm 796,74$ & $2500,75 \pm 310,51$ & $2303,75 \pm 921,57$ & $2402,50 \pm 608,63$ & $2493,25 \pm 780,74$ \\
\hline & I / ZEEP & $1968,75 \pm 796,75$ & $2666,00 \pm 670,96$ & $2505,25 \pm 1067,49$ & $2630,25 \pm 569,60$ & $2803,25 \pm 1446,94$ \\
\hline $\mathrm{ETCO}_{2}$ & H / PEEP & $44,75 \pm 2,5$ & $46,25 \pm 2,63^{A}$ & $45,50 \pm 2,38^{A}$ & $42,00 \pm 5,72^{A}$ & $39,00 \pm 5,12$ \\
\hline \multirow[t]{3}{*}{$\mathrm{mmHg}$} & H / ZEEP & $44,75 \pm 2,5^{a}$ & $41,00 \pm 2,45^{\mathrm{ABab}}$ & $39,50 \pm 1,73^{\mathrm{ABab}}$ & $38,50 \pm 1,73^{\mathrm{ABab}}$ & $43,00 \pm 6,68^{a b}$ \\
\hline & I / PEEP & $40,25 \pm 4,57$ & $40,75 \pm 7,14^{\mathrm{AB}}$ & $34,50 \pm 3,32^{\mathrm{B}}$ & $33,75 \pm 2,22^{\mathrm{B}}$ & $34,00 \pm 1,83$ \\
\hline & I / ZEEP & $40,25 \pm 4,58$ & $36,75 \pm 4,65^{\mathrm{Bb}}$ & $34,25 \pm 2,63^{\mathrm{Bb}}$ & $33,25 \pm 5,12^{\mathrm{Bb}}$ & $35,75 \pm 3,59^{b}$ \\
\hline
\end{tabular}

Valores expressos em média \pm desvio padrão onde: ${ }^{A B}=$ diferenças entre grupos ao longo dos momentos, ${ }^{\text {ab }}=$ diferenças entre tempos dentro de cada grupo, onde $\mathrm{p}<0,05 . \mathrm{SpO}_{2}=$ Saturação parcial de oxigênio na hemoglobina, $\mathrm{ETCO}_{2}=$ Concentração final de dióxido de carbono expirado; $\mathrm{FR}=$ Freqüência respiratória, $\mathrm{VC}=$ Volume corrente, VM = Volume minuto, H/PEEP = Grupo halotano + PEEP, H/ZEEP = Grupo halotano + ZEEP, I/PEEP = Isoflurano + PEEP, I/ZEEP = Isoflurano + ZEEP

A hipotensão foi observada desde a primeira mensuração, ou seja, nos valores basais, em todos os grupos e tempos, sendo maior nos pacientes que receberam halotano. O grupo que recebeu isoflurano e PEEP (I/PEEP) apresentou hipotensão gradativa, aumentando ao final dos 40 minutos. Podendo ser explicada por BERNARD et al. (1992) que observaram taquicardia e redução na pressão arterial média, em cães anestesiados com isoflurano e GILBERT et al. (1988) que reportaram menor redução da pressão arterial, do débito cardíaco e do volume ejetado com isoflurano quando comparado com a anestesia com halotano. $O$ isoflurano tem efeito mínimo sobre o sistema cardiovascular, atuando na manutenção do débito cardíaco em valores normais e apresenta efeito $\beta$-adrenérgico, causa diminuição da resistência vascular e hipotensão arterial e o halotano age diretamente sobre o miocárdio deprimindo o sistema cardiovascular (STEFEY, 1996).

A hipotensão observada pode ser explicada pela associação de vários fatores como o aumento da pressão intraabdominal ou intratorácica, o uso de agentes anestésicos halogenados, e também o uso da PEEP, pois todos estes fatores interferem no débito cardíaco e podem ter seus efeitos hipotensores potencializados quando associados.

MARCUS et al. (1996) observaram em suínos submetidos a pneumotórax experimental durante pneumoperitôneo aumento da PAS após insuflação intraabdominal e queda nesta variável após início do pneumotórax induzido, e retorno da pressão arterial a níveis basais após desinsuflação e aumento na FC após o inicio do pneumotórax. Neste experimento não foi observado elevação da PAS após 
insuflação abdominal e sim hipotensão, discordando dos achados do autor acima, porém os resultados concordam com MARCUS et al. (1996) no que se refere à maior hipotensão observada no grupo submetido a pneumoperitôneo em relação ao grupo submetido a pneumotórax.

TABELA 3 - FUNÇÃO HEMODINÂMICA DE SUÍNOS SUBMETIDOS A PNEUMOTÓRAX COM $5 \mathrm{mmHg}$ DE $\mathrm{CO}_{2}$, ANESTESIADOS COM HALOTANO OU ISOFLURANO E VENTILADOS COM PRESSÃO EXPIRATÓRIA FINAL POSITIVA DE $10 \mathrm{~cm}$ DE $\mathrm{H}_{2} \mathrm{O}$ (PEEP) OU PRESSÃO EXPIRATÓRIA FINAL ZERO (ZEEP) SANTA MARIA $\mathrm{RS} / 2002$.

\begin{tabular}{|c|c|c|c|c|c|c|}
\hline Variáveis & Grupos & Valores basais & $\begin{array}{r}10 \text { min de } \\
\text { ventilação }\end{array}$ & $\begin{array}{l}20 \text { min de } \\
\text { ventilação }\end{array}$ & $\begin{array}{c}30 \text { mim } \\
\text { ventilação }\end{array}$ & $\begin{array}{c}40 \text { min } \\
\text { ventilação }\end{array}$ \\
\hline PAS & H / PEEP & $86,00 \pm 24,99^{a}$ & $78,67 \pm 26,01^{a}$ & $66,00 \pm 20,07^{a b}$ & $69,67 \pm 21,36^{a b}$ & $57,67 \pm 8,96^{b}$ \\
\hline \multirow[t]{3}{*}{$\mathrm{mmHg}$} & $\mathrm{H} / \mathrm{ZEEP}$ & $86,00 \pm 24,99$ & $76,00 \pm 4,79$ & $71,25 \pm 17,76$ & $78,00 \pm 17,09$ & $68,50 \pm 16,13$ \\
\hline & I / PEEP & $85,75 \pm 16,40$ & $82,50 \pm 10,63$ & $87,50 \pm 10,63$ & $77,5 \pm 14,47$ & $68,25 \pm 16,50$ \\
\hline & I / ZEEP & $85,75 \pm 16,40$ & $78,50 \pm 35,07$ & $84,00 \pm 31,85$ & $84,75 \pm 23,08$ & $82,00 \pm 23,53$ \\
\hline PAM & H / PEEP & $75,00 \pm 22,18^{a}$ & $46,33 \pm 15,14^{b}$ & $43,67 \pm 20,82^{b}$ & $43,67 \pm 24,54^{b}$ & $36,00 \pm 6,24^{\mathrm{Bb}}$ \\
\hline \multirow[t]{3}{*}{$\mathrm{mmHg}$} & H / ZEEP & $75,00 \pm 22,18^{a}$ & $51,25 \pm 8,92^{\mathrm{ab}}$ & $45,75 \pm 11,18^{b}$ & $50,67 \pm 15,95^{a b}$ & $45,40 \pm 12,56^{\mathrm{ABab}}$ \\
\hline & I / PEEP & $61,75 \pm 10,53$ & $56,25 \pm 6,02$ & $64,75 \pm 4,99$ & $51,00 \pm 9,41$ & $50,00 \pm 13,11^{A B}$ \\
\hline & I / ZEEP & $61,75 \pm 10,53$ & $57,00 \pm 30,73$ & $54,50 \pm 23,58$ & $59,50 \pm 21,00$ & $59,00 \pm 20,14^{A}$ \\
\hline PAD & H / PEEP & $48,75 \pm 14,59^{a}$ & $35,00 \pm 11,27^{a b}$ & $30,67 \pm 15,01^{a b}$ & $33,00 \pm 19,97^{\mathrm{ab}}$ & $25,33 \pm 3,21^{b}$ \\
\hline \multirow[t]{3}{*}{$\mathrm{mmHg}$} & $\mathrm{H} / \mathrm{ZEEP}$ & $48,75 \pm 14,59$ & $39,25 \pm 9,54$ & $34,75 \pm 9,84$ & $34,00 \pm 15,52$ & $29,50 \pm 10,85$ \\
\hline & I / PEEP & $47,75 \pm 13,35$ & $42,50 \pm 9,74$ & $48,75 \pm 11,30$ & $35,5 \pm 7,23$ & $36,00 \pm 11,52$ \\
\hline & I / ZEEP & $47,75 \pm 13,35$ & $45,25 \pm 24,81$ & $43,50 \pm 22,75$ & $42,75 \pm 22,06$ & $42,50 \pm 22,86$ \\
\hline FC & $\mathrm{H} / \mathrm{PEEP}$ & $123,50 \pm 9,26{ }^{\mathrm{Aa}}$ & $88,50 \pm 7,77^{b}$ & $83,50 \pm 4,51$ & $85,50 \pm 4,43^{b}$ & $88,50 \pm 10,54^{b}$ \\
\hline \multirow[t]{3}{*}{ BPM } & $\mathrm{H} / \mathrm{ZEEP}$ & $123,50 \pm 9,26^{A}$ & $108,25 \pm 9,67$ & $102,75 \pm 19,48$ & $106,25 \pm 29,98$ & $103,00 \pm 23,69$ \\
\hline & I/ PEEP & $129,50 \pm 5,74^{\mathrm{Ba}}$ & $100,75 \pm 16,30^{b}$ & $100,75 \pm 16,30^{a b}$ & $104,75 \pm 13,1^{\mathrm{b}}$ & $94,50 \pm 11,36^{b}$ \\
\hline & I / ZEEP & $129,50 \pm 5,74^{\mathrm{Ba}}$ & $97,00 \pm 14,09^{b}$ & $103,75 \pm 24,40^{b}$ & $103,75 \pm 20,56^{b}$ & $110,25 \pm 25,32^{a b}$ \\
\hline \multirow[t]{4}{*}{ V\% } & $\mathrm{H} / \mathrm{PEEP}$ & $1,75 \pm 0,50^{A}$ & $1,50 \pm 0,46$ & $1,31 \pm 0,43^{\mathrm{A}}$ & $1,19 \pm 0,47^{\mathrm{A}}$ & $1,20 \pm 0,63^{A}$ \\
\hline & H / ZEEP & $1,75 \pm 0,50^{A}$ & $1,69 \pm 0,13$ & $1,75 \pm 0,20^{A}$ & $1,69 \pm 0,63^{A}$ & $1,63 \pm 0,63^{A}$ \\
\hline & I / PEEP & $3,25 \pm 0,50^{B}$ & $3,00 \pm 0,41$ & $3,00 \pm 0,41^{\mathrm{B}}$ & $3,00 \pm 0,00^{B}$ & $2,94 \pm 0,13^{\mathrm{B}}$ \\
\hline & I / ZEEP & $3,25 \pm 0,50^{\mathrm{Ba}}$ & $2,94 \pm 0,43^{b}$ & $2,88 \pm 0,48^{\mathrm{B}}$ & $2,88 \pm 0,48^{\mathrm{B}}$ & $2,88 \pm 0,48^{\mathrm{B}}$ \\
\hline
\end{tabular}

Valores expressos em média \pm desvio padrão onde: ${ }^{A B}=$ diferenças entre grupos ao longo dos momentos, ${ }^{\text {ab }}=$ diferenças entre tempos dentro de cada grupo, onde $p<0,05$ PAS $=$ Pressão arterial sistólica, $P A M=$ Pressão arterial média, $P A D=$ Pressão arterial diastólica, $\quad F C=$ Freqüência cardíaca, $V \%=$ Vaporização do gás anestésico, H/PEEP = Grupo halotano + PEEP, H/ZEEP = Grupo halotano + ZEEP, I/PEEP = Isoflurano + PEEP, I/ZEEP = Isoflurano + ZEEP.

Nos suínos submetidos a pneumoperitôneo não foi observada diferença entre grupos na freqüência cardíaca, mas houve uma redução de $35 \%$ em relação aos valores basais no grupo que recebeu halotano e $20 \%$ no grupo que recebeu isoflurano conforme TABELA 1. A freqüência cardíaca dos pacientes anestesiados com halotano e submetidos a PEEP manteve-se menor, quando comparada com os demais grupos. Já nos pacientes que receberam pneumotórax (TABELA 3) foi observado redução de $15 \%$ em relação aos valores basais na $\mathrm{FR}$, do grupo que recebeu halotano e $11 \%$ no grupo que recebeu isoflurano e ZEEP (I/ZEEP).

Também foi observada diferença significativa entre os valores basais e os demais tempos na freqüência respiratória (TABELAS 2 e 4) dos animais submetidos a pneumotórax e pneumoperitôneo. Acredita-se que estas diferenças se devem ao fato de que o plano anestésico destes pacientes encontrava-se mais superficial durante a primeira mensuração, em relação aos demais tempos, uma vez que neste período a anestesia dos mesmos estava sendo estabilizada. Talvez por este motivo os valores não variaram entre os demais tempos, uma vez que após o início da ventilação mecânica os animais permaneciam estabilizados por 80 minutos. 
TABELA 4 - FUNÇÃO RESPIRATÓRIA DE SUÍNOS SUBMETIDOS A PNEUMOTÓRAX COM 5 $\mathrm{mmHg}$ DE $\mathrm{CO}_{2}$, ANESTESIADOS COM HALOTANO OU ISOFLURANO E VENTILADOS COM PRESSÃO EXPIRATÓRIA FINAL POSITIVA DE $10 \mathrm{CM}$ DE $\mathrm{H}_{2} \mathrm{O}$ (PEEP) OU PRESSÃO EXPIRATÓRIA FINAL ZERO (ZEEP) SANTA MARIA $\mathrm{RS} / 2002$.

\begin{tabular}{|c|c|c|c|c|c|c|}
\hline Variáveis & Grupos & Valores basais & $\begin{array}{c}10 \text { min. de } \\
\text { ventilação }\end{array}$ & $\begin{array}{l}20 \text { min. de } \\
\text { ventilação }\end{array}$ & $\begin{array}{l}30 \text { mim. de } \\
\text { ventilação }\end{array}$ & $\begin{array}{l}40 \text { min. de } \\
\text { ventilação }\end{array}$ \\
\hline $\mathrm{SPO}_{2}$ & H/ PEEP & $98,25 \pm 1,26$ & $97,50 \pm 1,91^{A}$ & $96,50 \pm 5,74^{\mathrm{A}}$ & $99,50 \pm 1,00^{A}$ & $99,00 \pm 0,82^{A}$ \\
\hline \multirow[t]{3}{*}{$\%$} & H / ZEEP & $98,25 \pm 1,26$ & $85,25 \pm 8,54^{\mathrm{B}}$ & $91,75 \pm 10,72^{A}$ & $91,50 \pm 11,82^{\mathrm{B}}$ & $90,75 \pm 11,35^{\mathrm{B}}$ \\
\hline & I / PEEP & $97,25 \pm 2,21$ & $95,00 \pm 3,16^{A}$ & $96,75 \pm 1,70^{A}$ & $97,25 \pm 1,25^{\mathrm{A}}$ & $97,50 \pm 1,29^{A}$ \\
\hline & I / ZEEP & $97,25 \pm 2,2$ & $85,00 \pm 15,40^{B}$ & $87,75 \pm 12,03^{B}$ & $88,75 \pm 11,67^{B}$ & $89,50 \pm 11,15^{B}$ \\
\hline FR & H / PEEP & $15,50 \pm 5,80^{a}$ & $11,75 \pm 2,63^{b}$ & $12,00 \pm 2,94^{b}$ & $10,25 \pm 2,06^{b}$ & $10,50 \pm 1,73^{b}$ \\
\hline \multirow[t]{3}{*}{ MPM } & $\mathrm{H} / \mathrm{ZEEP}$ & $15,50 \pm 5,80^{a}$ & $13,00 \pm 4,40^{b}$ & $10,50 \pm 1,73^{b}$ & $11,00 \pm 2,16^{b}$ & $12,50 \pm 3,70^{b}$ \\
\hline & I / PEEP & $17,75 \pm 3,10$ & $12,50 \pm 2,38$ & $12,50 \pm 2,38$ & $10,75 \pm 2,50$ & $10,50 \pm 1,91$ \\
\hline & I / ZEEP & $17,75 \pm 3,10^{a}$ & $13,25 \pm 2,36^{b}$ & $13,25 \pm 2,75^{\mathrm{b}}$ & $11,75 \pm 2,36^{b}$ & $13,00 \pm 2,16^{b}$ \\
\hline VC & H / PEEP & $158,75 \pm 143,87$ & $188,75 \pm 45,98$ & $186,00 \pm 40,21$ & $191,75 \pm 74,46$ & $174,33 \pm 17,79$ \\
\hline \multirow[t]{3}{*}{$\mathrm{ml}$} & $\mathrm{H} / \mathrm{ZEEP}$ & $158,75 \pm 143,87$ & $172,00 \pm 86,27$ & $228,75 \pm 84,01$ & $189,50 \pm 60,12$ & $181,50 \pm 74,68$ \\
\hline & I / PEEP & $133,25 \pm 30,58^{b}$ & $161,75 \pm 81,88^{a}$ & $161,75 \pm 81,88^{a}$ & $211,25 \pm 81,57^{\mathrm{a}}$ & $195,50 \pm 66,57^{a}$ \\
\hline & I / ZEEP & $133,25 \pm 30,55^{b}$ & $170,00 \pm 35,11^{a}$ & $179,50 \pm 36,92^{a}$ & $184,00 \pm 58,01^{a}$ & $174,50 \pm 77,76^{a}$ \\
\hline VM & H / PEEP & $1825,75 \pm 440,55$ & $1858,00 \pm 613,97$ & $2241,75 \pm 194,73$ & $1964,75 \pm 321,46$ & $1922,00 \pm 218,42$ \\
\hline \multirow[t]{3}{*}{$\mathrm{ml} / \mathrm{min}$} & H / ZEEP & $1825,75 \pm 440,55$ & $2084,25 \pm 485,79$ & $1457,00 \pm 3007,00$ & $2008,25 \pm 350,53$ & $2107,00 \pm 292,68$ \\
\hline & I / PEEP & $1952,25 \pm 784,96^{b}$ & $1961,75 \pm 1323,85^{a b}$ & $1961,75 \pm 1323,85^{a}$ & $2356,50 \pm 1352,79^{a b}$ & $2143,50 \pm 973,06^{a b}$ \\
\hline & I / ZEEP & $1952,25 \pm 784,96$ & $2096,50 \pm 567,73$ & $2360,75 \pm 833,35$ & $1905,25 \pm 626,66$ & $2144,00 \pm 1266,81$ \\
\hline $\mathrm{ETCO}_{2}$ & $\mathrm{H} / \mathrm{PEEP}$ & $40,50 \pm 5,32$ & $39,00 \pm 2,94$ & $35,75 \pm 2,63$ & $37,50 \pm 2,65$ & $38,75 \pm 4,65$ \\
\hline \multirow[t]{3}{*}{$\mathrm{mmHg}$} & $\mathrm{H} / \mathrm{ZEEP}$ & $40,50 \pm 5,32$ & $47,00 \pm 11,94$ & $39,50 \pm 11,09$ & $40,50 \pm 9,71$ & $43,00 \pm 12,03$ \\
\hline & I / PEEP & $42,75 \pm 8,30$ & $47,00 \pm 8,68$ & $47,00 \pm 8,68$ & $36,25 \pm 4,11$ & $36,50 \pm 3,42$ \\
\hline & I / ZEEP & $42,75 \pm 8,30$ & $39,50 \pm 4,93$ & $42,75 \pm 6,34$ & $42,50 \pm 4,51$ & $4300, \pm 4,24$ \\
\hline
\end{tabular}

Valores expressos em média \pm desvio padrão onde: ${ }^{\mathrm{AB}}=$ diferenças entre grupos ao longo dos momentos, ${ }^{\mathrm{ab}}=$ diferenças entre tempos dentro de cada grupo, onde $\mathrm{p}<0,05 \quad \mathrm{SpO}_{2}=$ Saturação parcial de oxigênio na hemoglobina, $\mathrm{ETCO}_{2}=$ Concentração final de dióxido de carbono expirado, $\mathrm{FR}=$ Freqüência respiratória, $\mathrm{VC}=$ Volume corrente, $\mathrm{VM}=$ Volume minuto, H/PEEP $=$ Grupo halotano $+\mathrm{PEEP}, \mathrm{H} / \mathrm{ZEEP}=$ Grupo halotano + ZEEP I/PEEP = Isoflurano + PEEP, I/ZEEP = Isoflurano + ZEEP.

Segundo CHRISTOPHER et al. (1999) quando se observa uma alteração na $\mathrm{ETCO}_{2}$ imediatamente se procura ajustar o volume-minuto, para manter os níveis de $\mathrm{CO}_{2}$ estáveis. Neste experimento a $\mathrm{ETCO}_{2}$ foi mantida entre 35 e $45 \mathrm{~mm} \mathrm{Hg}$, ou seja, em níveis fisiológicos, com a intenção de manter a eliminação do $\mathrm{CO}_{2}$ constante. Por este motivo não foi observada diferença significativa entre tempos nas variáveis $\mathrm{FR}, \mathrm{VC}, \mathrm{VM}, \mathrm{ETCO}_{2}$, (TABELAS 2 e 4), indicando adequada ventilação durante todo o experimento. GUYTON (1991) relata que a diferença entre pressão de $\mathrm{CO}_{2}$ alveolar $\mathrm{e}$ sanguínea é mínima, cerca de $1 \mathrm{~mm} \mathrm{Hg}$, devido à rápida difusão através das membranas celulares, sendo bastante complicado a mensuração desta diferença, assim a ETCO2 é um excelente parâmetro para monitorar pacientes onde se visa normocapnia.

Os pacientes foram anestesiados com $2,5 \mathrm{~V} \%$ de isoflurano concordando com THURMON (1996) que relataram que a manutenção anestésica com isoflurano deve ser de 2,0 a 3,0V\%. Entretanto a concentração de $0,88 \mathrm{~V} \%$ de halotano utilizada neste experimento difere dos valores de 1,5 a $2,5 \mathrm{~V} \%$ citados para o halotano pelo mesmo autor.

\section{Conclusão}

A ventilação mecânica com PEEP de 10 $\mathrm{cm} \mathrm{H}_{2} \mathrm{O}$ produz valores de $\mathrm{SpO}_{2}$ superiores à ZEEP e aumenta a hipotensão desencadeada pela associação dos efeitos da anestesia geral com halotano ou isoflurano e os efeitos do pneumotórax ou do pneumoperitôneo sobre a hemodinâmica. 


\section{Referências}

BAILEY, J.E.; PABLO, L.S. Anesthetic and physiologic considerations for veterinary endosurgery In: FREEMAN, L. J. Veterinary Endosurgery. Boston: Mosby, 1998. p. 24-43.

BERNARD, J.M.; DOURSOUT, M.F.; WOUTERS, P.; HARTLEY, C.J.; MERIN, R.G.; CHELLY, J.E. Effects of Sevoflurane and Isoflurane on Hepatic Circulation in the Chronically Instrumented Dog. Anesthesiology, Hagerstown, v. 77, n. 3, p. 541-545, 1992.

BROCK, H.; RIEGER, R.; GABRIEL, C.; POLZ, W.; MOOSBAUER, W.; NECEK, S. Hemodynamic changes during toracoscopic surgery: The effects of one-lung ventilation compared with carbon dioxide insufflation. Anesthesia, v. 55, n. 1, p. 10-16, 2000.

CHRISTOPHER, S.N.G.; GILL, I.S.; SUNG, G.T.; WHALLEY, D.G.; GRAHAM, R.; SCHWEIZER, D. Retroperitoneoscopic surgery is not associated with increased carbon dioxide absorption. Journal of Urology, Baltimore, v. 162 , n. 4, p. 1268,1999

GILBERT, M.; ROBERTS, M.D.; MORI, M.; BLOMBERG, R.; TINKER, J.H. Comparative Coronary Reactivity and Hemodynamic During Halothane and Isoflurane Anesthesia in Swine. Anesthesiology, Hagerstown, n. 68, p. 243253, 1988

GUYTON, A.C. Tratado de Fisiologia Médica. Rio de Janeiro: Guanabara-Koogan, 1991. p. 351-406.

JONES, D.R.; GRAEBER, G.M.; TANGUILIG, G.G.; MURRIA, G.F. Effects of insufflations on hemodynamic during thoracoscopy. Annals of Thoracic Surgery, New York, v. 55, n. 6, p. 1379-1382, 1993.

LOECKINGER, A.; KLEINSASSER, A.; HOERMANN, C.; ANETTE, K.; GASSNER, M.; FRIEDRICH, P.; LINDNER, K. Inert Gas Exchange During Pneumoperitoneum at Incremental Values of Positive End-Expiratory Pressure. Anesthesia \& Analgesia, Baltimore, v. 90 p. 466-472, 2000.

Recebido para publicar: $\quad$ 25/06/2002 Aprovado:
MALLEY, W.J. Clinical Blood Gases, Application and Noninvasive Alternatives. Philadelphia: Saunders, 1990. 379p.

MARCUS, D.R.; LAU, W.M.; SWANSTROM, L.L. Carbon Dioxide Pneumothorax in Laparoscopic Surgery. The American Journal of Surgery, Desplaines, v. 171, p. 464-466, 1996.

OHTSUKA, T.; IMANAKA, K.; ENDOH, M.; KOHNO, T.; NAKAJIMA, J.; KOTSUKA, Y. Hemodynamic Effects of Carbon Dioxide Insuffation Under Single-Lung Ventilation During Thoracoscopy. Annals of Thoracic Surgery, New York, v. 68, p. 29-33, 1999.

PADDLEFORD, R.R. Manual of small Animal Anesthesia 2. Ed. Philadelphia: Saunders, 1999.

PAW, P.; SACKIER, J.M. Complications of laparoscopy and thoracoscopy. Journal of Intensive Care Medicine, Cambridge, v. 6, n. 9, p. 290-304, 1994.

STEFEY, E.P. Inhalation Anesthetics. In: THURMON, J.C.; TRANQUILLI, W.J.; BENSON, G.J. Lumb \& Jones Veterinary Anesthesia, Philadelphia: Williams \& Wilkins, 1996. p.297-299.

THURMON, J.C.; TRANQUILLI, W.J.; BENSON, G.J. Lumb \& Jones Veterinary Anesthesia. Philadelphia: Williams \& Wilkins, 1996. $928 \mathrm{p}$.

TOGAL, T.; GULHAS, N.; CICEK, M. Carbon dioxide pneumothorax during laparoscopic surgery. Surgery Endoscopic, Berlin, v. 4, p. 123-128, 2002.

TORRES, M.L.A.; BONASSA, J. Anestesia Inalatória. In: FANTONI, D.T.; CORTOPASSI, S.R.G. Anestesia em Cães e Gatos. São Paulo:Roca, 2002. p.95-106.

WALSH, P.J.; REMEDIOS, A.M.; FERGUSON, J.F. Thoracoscopic versus open pericardlectomy in dogs: Comparison of postoperative pain and morbidity. Veterinary Surgery, Philadelphia, v. 28 , p. $472-479$, 1999. 